\title{
Frame Error Probability Analysis of Mobile Underwater Acoustic Networks
}

\author{
Andrej Stefanov
}

\begin{abstract}
The paper analyzes the frame error probability (FEP) of mobile underwater acoustic networks. The mobility model is direction persistent: the direction and the speed of the autonomous underwater vehicles (AUV's) are constant for the duration of the packet. The frame error probability is evaluated using frequency dependent path loss and Ricean fading for each AUV-to-AUV channel. The FEP performance is illustrated through numerical examples.
\end{abstract}

Keywords—underwater acoustic networks, mobility.

\section{Introduction}

There have been significant recent research efforts focused on the analysis and design of underwater networks [1-4], including medium access control and routing protocols, see, e.g., [5-7] and references therein. The study of mobile underwater acoustic networks is also of particular interest as a network of AUV's may be an appealing choice for sensing and surveying of a given area for environmental, scientific, and/or commercial objectives.

The paper studies the FEP of mobile underwater acoustic networks. The reserve listen and go transmission protocol along the multihop route from the source to the destination is considered. In particular, the focus is on an idealized scenario where the protocol ensures that the impact of potential interference is essentially negligible. The mobility model is direction persistent, that is, the direction and the speed of the AUV's are constant for the duration of the packet. Each AUV-to-AUV link is characterized by the frequency dependent path loss model and independent Ricean fading.

The paper is organized as follows. The underwater acoustic propagation model is given in Sections II. The FEP performance based on the reserve listen and go transmission protocol and the direction persistent mobility model is evaluated in Section III. Numerical examples are given in Section IV. Section V concludes the paper.

Andrej Stefanov

Faculty of Engineering

IBU Skopje

Macedonia

\section{Underwater Acoustic Propagation}

Underwater acoustic propagation experiences a path loss, which at distance $d$ between the transmitter and the receiver, and for a signal transmitted on frequency $f$, is

$$
A(d, f)=A_{0} d^{\kappa} a(f)^{d}
$$

where $A_{0}$ is a unit-normalizing constant that incorporates fixed losses, $a(f)$ is the absorption coefficient and $\kappa$ is the spreading factor $(1 \leq \kappa \leq 2)$. For practical spreading, $\kappa=1.5$. Using Thorp's formula which gives $a(f)$ in $\mathrm{dB} / \mathrm{km}$ for $f$ in $\mathrm{kHz}$, the absorption coefficient is

$$
10 \log a(f)=\frac{0.11 f^{2}}{1+f^{2}}+\frac{44 f^{2}}{4100+f^{2}}+\frac{2.75 f^{2}}{10^{4}}+0.003
$$

This formula is typically valid for frequencies above several hundred $\mathrm{Hz}$.

The ocean ambient noise is modeled as composed of turbulence, shipping, waves and thermal noise, described by Gaussian statistics and continuous power spectral densities (p.s.d.'s). The respective noise components have formulae that give their p.s.d.'s in $\mathrm{dB}$ re $\mu \mathrm{Pa}$ per $\mathrm{Hz}$ as a function of frequency in $\mathrm{kHz}$ [8]:

$$
\begin{gathered}
10 \log N_{\mathrm{t}}(f)=17-30 \log f, \\
10 \log N_{\mathrm{s}}(f)=40+20(s-0.5)+26 \log f \\
-60 \log (f+0.03), \\
10 \log N_{\mathrm{w}}(f)=50+7.5 \sqrt{w}+20 \log f \\
-40 \log (f+0.4), \\
10 \log N_{\mathrm{th}}(f)=-15+20 \log f,
\end{gathered}
$$

where $s$ is the shipping activity factor and $w$ is the wind speed in $\mathrm{m} / \mathrm{s}$. The total p.s.d. of the ambient noise is

$$
N(f)=N_{\mathrm{t}}(f)+N_{\mathrm{s}}(f)+N_{\mathrm{w}}(f)+N_{\mathrm{th}}(f) .
$$

The narrow-band signal-to-noise ratio (snr) at distance $d$, for a signal with p.s.d. $S(f)$, is

$$
\operatorname{snr}(d, f)=\frac{s(f)}{A(d, f) N(f)} .
$$

Figure 1 depicts $1 / A(d, f) N(f)$ which illustrates the narrow-band snr dependence on the frequency and the distance. It can be readily observed that for each distance there is a preferred operating frequency. 


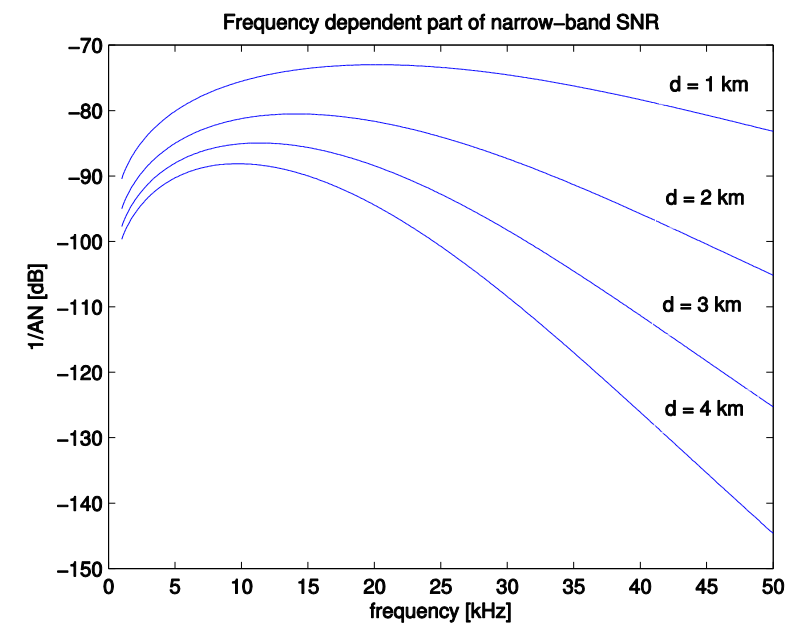

Fig. 1. The AN product for different distances $(\kappa=1.5)$.

\section{Mobile Network Setup}

The section considers the FEP for mobile underwater acoustic networks in the context of the reserve listen and go transmission protocol and the direction persistent mobility model.

\section{A. Transmission Protocol}

It is assumed that the network utilizes a reserve listen and go transmission protocol along the multihop route from the source to the destination [9]. Before transmitting the AUV first senses the channel in order to determine if it is idle. It starts transmitting only in the case that the channel is idle. Otherwise, if it determines that there is another transmission by another multihop route, the transmission is delayed. In an idealized scenario, the protocol ensures that the impact of potential interference is essentially negligible. The FEP performance is therefore basically determined by the performance in the idealized case when there is no interference in the network.

\section{B. Mobility Model}

We consider $N$ AUV's deployed over a network with circular area $\mathcal{A}$. The density of AUV's is $\rho=N / \mathcal{A}$. It is assumed that all AUV's are confined/deployed to the network area, that is, no AUV's enter and/or leave the network. In other words, the density of the network is constant. This could be a suitable model for a network of AUV's that are required to survey a certain area.

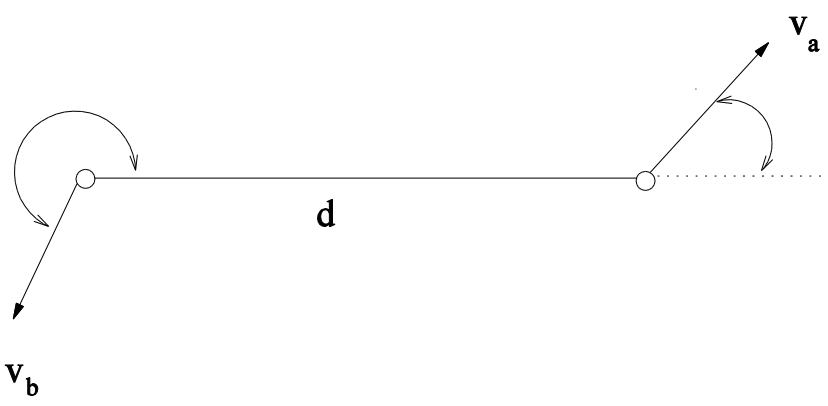

Fig. 2. AUV's: time $=t$.

The mobility status of an AUV is characterized by its speed and direction angle. Note that it is assumed that links along the route are independent from one another with respect to the AUV's mobility. In other words, the mobility status of an AUV during the packet reception is independent from the mobility status of that AUV during the packet transmission on the next hop along the route. The direction persistent mobility model means that the direction and the speed of the AUV's are constant for the duration of the packet.

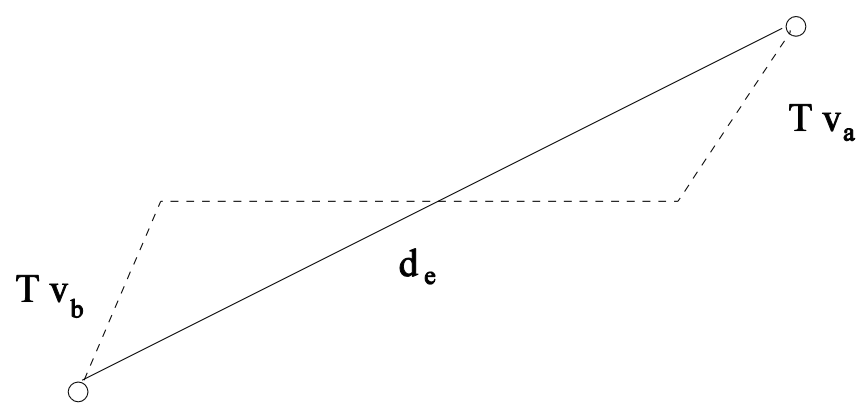

Fig. 3. AUV's: time $=t+T$.

The distance between AUV's at time $t$ is $d$. AUV $a$ is moving with speed $v_{a}$ in the direction given by the angle $\theta_{a}$ (the angle between $v_{a}$ and the horizontal axis). AUV $b$ is moving with speed $v_{b}$ in the direction given by the angle $\theta_{b}$. At time $t+T$ the distance between the AUV's is [9]

$$
d_{e}=\left(d^{2}+T^{2} u_{1}-2 T^{2} u_{2}+2 d T u_{3}\right)^{1 / 2}
$$

where

$$
\begin{array}{lc}
u_{1}= & v_{a}^{2}+v_{b}^{2}, \\
u_{2}= & v_{a} v_{b} \cos \left(\theta_{a}-\theta_{b}\right), \\
u_{3}= & v_{a} \cos \left(\theta_{a}\right)-v_{b} \cos \left(\theta_{b}\right) .
\end{array}
$$


The time interval $T$ is given by

that is,

$$
T=T_{\text {packet }}+T_{\text {propagation }}
$$

$$
T=\frac{L}{R_{b}}+\frac{d}{c}
$$

where $L$ is the number of bits per packet, $R_{b}$ is the bit rate in bits per second, and $c=1500 \mathrm{~m} / \mathrm{s}$ is the propagation speed of sound underwater.

The average distance between AUV's is

$$
\bar{d}=\frac{d+d_{e}}{2} .
$$

\section{Average Route FEP}

It is assumed that AUV's utilize simple demodulate and forward relaying strategy. The end-to-end route FEP is

$$
\mathrm{FEP}_{\text {route }}=1-\left(1-p_{b}\right)^{L n_{h}}
$$

where $p_{b}$ is the bit error probability of an AUV-to-AUV link, and $n_{h}$ is the number of hops along the multihop route. Considering a large number of realizations over $(v, \theta)$, the ensemble averaged $\mathrm{FEP}_{\text {route }}$ is

$$
\overline{\mathrm{FEP}_{\text {route }}}=\frac{\sum_{m=1}^{M} \mathrm{FEP}_{\text {route }}}{M}
$$

which can be evaluated through Monte Carlo simulation provided a sufficiently large number of realizations is considered. In particular, the focus is on the end-to-end frame error probability of a multihop route with an average number of hops, $\overline{n_{h}}=\sqrt{N / \pi}$ [9].

Without the loss of generality, we focus on uncoded BPSK transmission [10]. Under the assumption of a Ricean fading model for the AUV-to-AUV channel [11], and assuming that perfect channel state information is available at the receiver, the bit error probability is

$$
p_{b} \leq \frac{1+\mathcal{K}}{1+\mathcal{K}+\operatorname{snr}(\bar{d}, f)} \cdot \exp \left(-\frac{\mathcal{K} \operatorname{snr}(\bar{d}, f)}{1+\mathcal{K}+\operatorname{snr}(\bar{d}, f)}\right)
$$

where $\mathcal{K}$ is the Ricean fading parameter which is assumed to be the same for all AUV-to-AUV links. The attenuation and noise are assumed to be constant over the operational bandwidth, so that the snr at the operating frequency $f_{o}(\bar{d})$ is

$$
\operatorname{snr}\left(\bar{d}, f_{o}\right)=\frac{P}{A\left(\bar{d}, f_{o}\right) N\left(f_{o}\right) B^{\prime}}
$$

where $B$ is the bandwidth and $P$ is the transmit power. This is a suitable approximation for the narrow bandwidth case or the sub-band of one carrier in a multi-carrier system, e.g., OFDM [12].

\section{Numerical Results}

Next, we present numerical examples that illustrate the average route FEP of a mobile underwater acoustic network based on the considered approach. The focus is on the FEP of a multihop route with an average number of hops. It is averaged over $M=1000$ realizations. The circular network area is $\mathcal{A}=1000 \mathrm{~km}^{2}$. Independent Ricean fading for each AUV-to-AUV channel with $\mathcal{K}=10$ is assumed. Fixed losses are neglected [4]. The frame size is $L=1000$ bits. The bit rate is $R_{b}=1 \mathrm{kbps}$. It is assumed that all AUV's operate with the same transmit power level. The AUV's are assumed to move at a speed of $v=1 \mathrm{~m} / \mathrm{s}$. The spreading factor is $\kappa=1.5$, the shipping activity factor is $s=0.5$, and unless otherwise indicated, the wind speed is $w=0$.

Figure 4 presents the route FEP for different nodes transmit powers. Powers $P=115 \mathrm{~dB}$ re $\mu \mathrm{Pa}, P=120 \mathrm{~dB}$ re $\mu \mathrm{Pa}$ and $P=125 \mathrm{~dB}$ re $\mu \mathrm{Pa}$ are considered. The bandwidth is $B=4 \mathrm{kHz}$. It can be observed that the performance improves with an increase in the transmit power levels. $P=125 \mathrm{~dB}$ re $\mu \mathrm{Pa}$ can provide $\mathrm{FEP}_{\text {route }}=10^{-3}$. Note that this is due to the consideration of an idealized version of the reserve listen and go transmission protocol which ensures that AUV's transmissions are not impacted by interference.

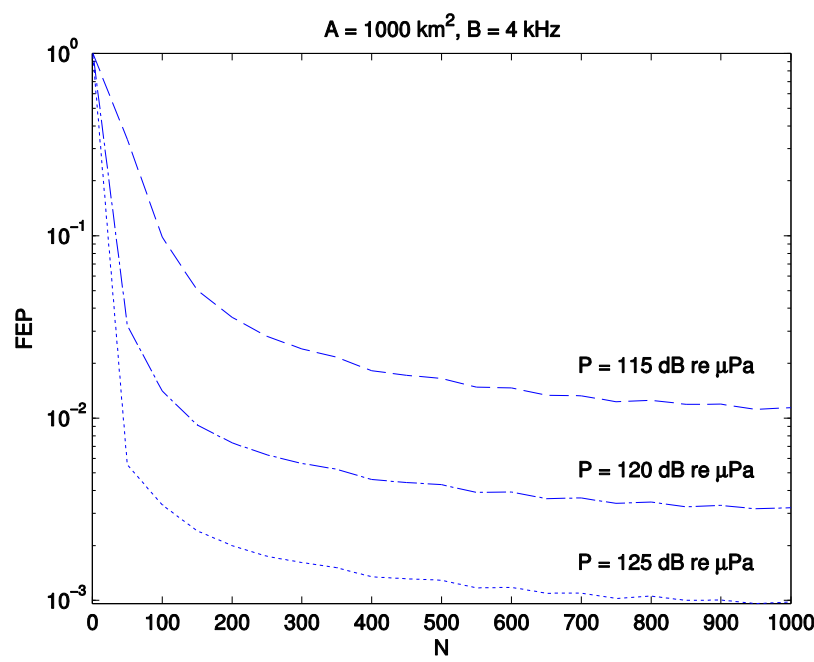

Fig. 4. FEP for different powers.

Figure 5 illustrates the route FEP for different bandwidths. Bandwidths $B=4 \mathrm{kHz}, B=8 \mathrm{kHz}$, and $B=12 \mathrm{kHz}$ are considered. The transmit power is $P=125 \mathrm{~dB}$ re $\mu \mathrm{Pa}$. It can be seen that the increase in bandwidth results in an increase of the route FEP. This is due to the decrease in the snr, since the noise power increases with the increase in bandwidth while the transmit power remains constant. 


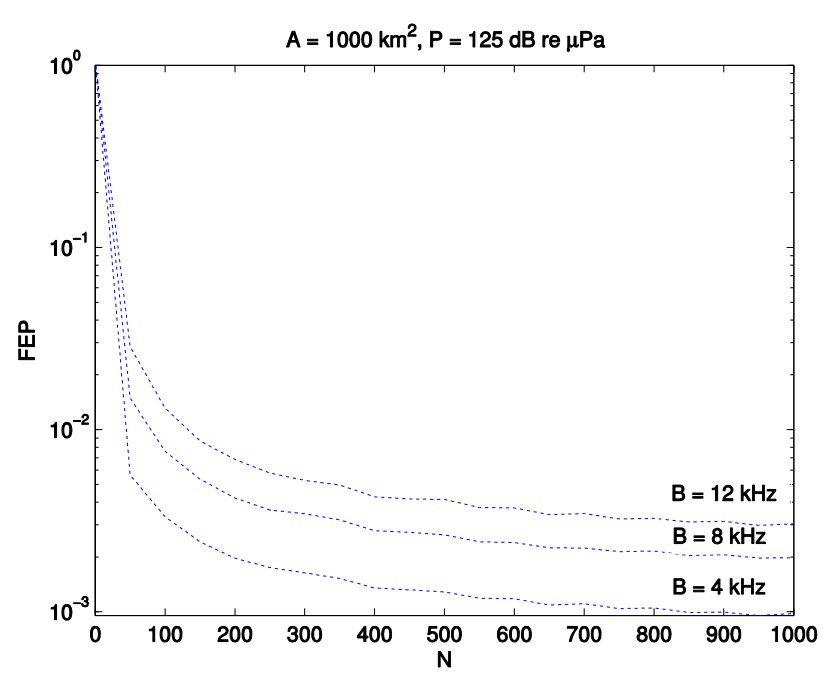

Fig. 5. FEP for different bandwidths.

Figure 6 depicts the route FEP for different wind speeds. The transmit power is $P=125 \mathrm{~dB}$ re $\mu \mathrm{Pa}$. The bandwidth is $B=4 \mathrm{kHz}$. When there is no wind, that is, $w=0 \mathrm{~m} / \mathrm{s}$, the route FEP can achieve $\mathrm{FEP}_{\text {route }}=10^{-3}$. However, as the wind speed increases to $w=2 \mathrm{~m} / \mathrm{s}$ and $w=4 \mathrm{~m} / \mathrm{s}$, it can be observed that the route FEP remains above $10^{-2}$.

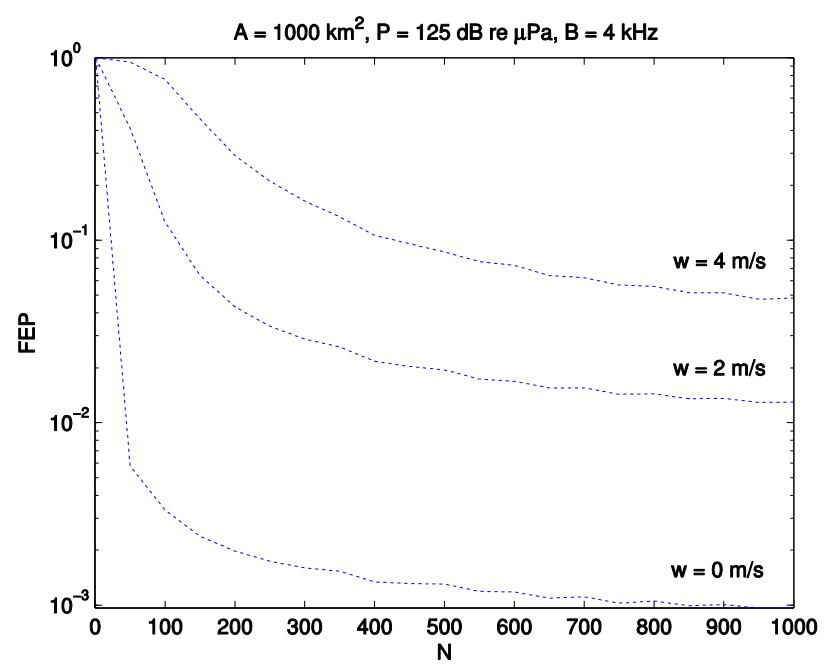

Fig. 6. FEP for different wind speeds.

\section{v. Conclusions}

The paper considered the FEP performance of mobile underwater acoustic networks. The focus was on the evaluation of the average route FEP in the context of the reserve listen and go transmission protocol and the direction persistent mobility model. It was assumed that the number of AUV's in the network area remains constant, in other words, no AUV's enter and/or leave the network. Additionally, it was assumed that there is frequency dependent path loss and Ricean fading for each AUV-toAUV channel. Numerical examples illustrated how the average route FEP of the mobile network depends on different transmit powers, bandwidths, as well as different wind speeds.

\section{References}

[1] Special Issue on Underwater Wireless Communications and Networks, IEEE Journal Sel. Areas Commun., vol. 26, Dec. 2008.

[2] Special Issue on Underwater Networks, Ad Hoc Networks, vol. 7, Elsevier, Jun. 2009.

[3] Special Issue on Underwater Sensor Nodes and Underwater Sensor Networks, Sensors, MDPI Publishing, 2011-2012.

[4] A. Stefanov and M. Stojanovic, "Design and Performance Analysis of Underwater Acoustic Networks," IEEE Journal Sel. Areas Commun., vol. 29, pp. 2012-2021, Dec. 2011.

[5] K. Chen, M. Ma, E. Cheng, F. Yuan, and W. Su, "A Survey on MAC Protocols for Undewater Wireless Sensor Networks," IEEE Communications Surveys and Tutorials, vol. 16, pp. 1433-1447, Mar. 2014.

[6] H.-H. Cho, C.-Y. Chen, T.-K. Shih, H.-C. Chao, "Survey on Underwater Delay/Disruption Tolerant Wireless Sensor Network Routing," IET Wireless Sensor Systems, vol. 4, pp. 112-121, Sep. 2014.

[7] N. Li, J.-F. Martinez, J. M. M. Chaus, and M. Eckert, "A Survey on Underwater Acoustic Sensor Network Routing Protocols," Sensors, vol. 16, MDPI Publishing, Mar. 2016.

[8] L. Berkhovskikh and Y. Lysanov, "Fundamentals of Ocean Acoustics," Springer, 1982.

[9] O. Tonguz and G. Ferrari, "Ad Hoc Wireless Networks: A Communication-Theoretic Perspective," Wiley, 2006.

[10] S. Benedetto and E. Biglieri, "Principles of Digital Transmission with Wireless Applications," Kluwer/Plenum, 1999.

[11] P. Qarabagi and M. Stojanovic, "Statistical Characterization and Computationally Efficient Modeling of a Class of Underwater Acoustic Communication Channels," IEEE Journal of Oceanic Engineering, vol. 38, pp. 701-717, Oct. 2013.

[12] L. Hanzo and T. Keller, "OFDM and MC-CDMA: A Primer," Wiley-IEEE Press, 2006. 